\title{
Imaging Lung Disease in Systemic Sclerosis
}

\author{
Diane Strollo • Jonathan Goldin
}

Published online: 16 March 2010

(C) The Author(s) 2010. This article is published with open access at Springerlink.com

\begin{abstract}
Interstitial lung disease and pulmonary hypertension $(\mathrm{PH})$ are the most common cardiopulmonary findings in patients with systemic sclerosis (SSc). About two thirds of patients suffering from SSc develop scleroderma interstitial lung disease. $\mathrm{PH}$ is present in about $20 \%$ of SSc patients and is typically associated with severe lung disease, although it may be an isolated manifestation of SSc. High-resolution CT scanning is a key method for evaluating chest involvement. There are four roles of imaging in scleroderma interstitial lung disease: 1) detection of lung involvement, 2) identification of patients likely to respond to treatment, 3) assessment of treatment efficacy, and 4) exclusion of other significant diseases to include $\mathrm{PH}$ and cardiac and esophageal abnormalities.
\end{abstract}

Keywords Scleroderma. CT lung disease review

\section{Introduction}

Systemic sclerosis (SSc), or scleroderma, is a chronic multisystem disorder of small vessels and connective tissues characterized by diffuse fibrosis of the skin and internal organs, most frequently the lungs and gastrointestinal tract. The disease has a 3:1 female predilection and

\section{Strollo}

Department of Radiology, University of Pittsburgh Medical Center, 200 Lothrop St, PUH, E-177, Pittsburgh, PA 15213, USA

e-mail: strollode@upmc.edu

J. Goldin $(\bowtie)$

Department of Radiology,

David Geffen School of Medicine at UCLA,

10833 Le Conte Avenue,

Los Angeles, CA 90095-1721, USA

e-mail: jgoldin@mednet.ucla.edu typically occurs in the third to fifth decades of life. Almost all patients have skin involvement. Up to $80 \%$ of patients have limited cutaneous disease, which typically has an insidious onset and skin changes confined to the face and extremities, whereas $20 \%$ with diffuse involvement may have more severe and rapidly progressive skin, lung, cardiac, and gastrointestinal disease [1]. Patients with interstitial lung disease (ILD) and pulmonary vascular disease may be severely symptomatic due to restrictive lung physiology and low diffusing capacity. Esophageal dysmotility occurs in almost all SSc patients, and gastroesophageal reflux may mimic or overlap with ILD. Cardiac involvement, to include right heart dysfunction due to pulmonary hypertension (PH), myocardial fibrosis, and pericardial involvement, may further compound pulmonary symptoms.

ILD and $\mathrm{PH}$ are the most common cardiopulmonary findings in SSc $[2,3]$. About two thirds of patients suffering from SSc develop scleroderma ILD (SILD) [4-6]. PH is present in about $20 \%$ of SSc patients and is typically associated with severe pulmonary disease, although it may be an isolated manifestation of SSc [2]. Currently, SILD is the leading cause of death in SSc patients, due to pulmonary fibrosis with or without $\mathrm{PH}$. The mortality rate from SILD is about $40 \%$ within 10 years after the onset of the disease [7-9].

There are four key roles of imaging in ILD related to SSc: 1) detection of lung involvement, 2) identification of patients likely to respond to treatment, 3) assessment of treatment efficacy, and 4) exclusion of other significant diseases to include $\mathrm{PH}$ and cardiac and esophageal abnormalities.

\section{Detection}

Chest radiography is highly insensitive in detecting and assessing the extent of lung involvement in SSc patients 
[10]. In fact, patients with pulmonary symptoms and early lung involvement may have a normal chest radiograph. Radiographic findings of SILD are observed in up to two thirds of symptomatic patients, whereas pulmonary fibrosis is detected in only $25 \%$ to $44 \%$ [11]. SILD typically manifests as low lung volume and predominantly increased ground glass opacity (GGO) and reticular interstitial thickening that is greatest in the lung bases. Advanced cases with pulmonary fibrosis may exhibit traction bronchiectasis and honeycomb cystic change. However, some patients with pulmonary fibrosis may have subtle radiographic findings $[10,11]$.

High-resolution CT (HRCT) has been shown to be more accurate than chest radiography in detecting and characterizing diffuse lung diseases, and abnormalities on CT correlate more closely with pulmonary function test (PFT) abnormalities [12-14]. HRCT is now well-established as a sensitive and noninvasive means of detecting and characterizing SILD [15-19]. CT features of fibrosis are present in $55 \%$ to $65 \%$ of all patients with SSc and in up to $96 \%$ of those with abnormal PFT results $[5,11,13,14,17,19,20]$. As a result, HRCT has become an important part of the routine evaluation of SILD. Classically, the disease affects juxtapleural, posterior, and basilar portions of the lungs, with initially subtle alterations of increased GGO, defined as increased lung attenuation in the absence of architectural distortion, as well as accentuated reticular markings that may progress to pulmonary fibrosis, defined as architectural distortion with reticular intralobular interstitial thickening, traction bronchiectasis and bronchiolectasis, and honeycomb cystic change (Figs. 1 and 2). These hallmark CT features of SILD are similar to those of idiopathic, nonspecific interstitial pneumonitis (NSIP). A similar association has been reported in radiologic-pathologic correlations of surgical lung biopsy specimens of patients with SSc [20-23].
SILD typically manifests on $\mathrm{CT}$ as predominantly GGO with an admixture of pulmonary fibrosis consistent with the NSIP pattern. Honeycomb cystic changes are reported in $11 \%$ to $37 \%$ of patients with SILD. This is unlike other patients with NSIP, who have little or no cystic change [23-25]. As honeycomb cystic change is typically a marker for usual interstitial pneumonia (UIP) and pulmonary fibrosis [26], these findings suggest that patients with SILD disease may have a mixture (or overlap) of UIP and NSIP patterns. This is consistent with the findings of Desai et al. [16] and other autopsy studies, in which typical histopathologic findings of SILD included marked pulmonary fibrosis and honeycomb cystic change [24, 27, 28].

It has been incorrectly assumed that SILD is more common and severe in patients with diffuse cutaneous systemic sclerosis $(\mathrm{dcSSc})$. In several recent studies, about $40 \%$ of patients with pulmonary involvement have demonstrated cutaneous systemic sclerosis (lcSSc) $[8,9,29,30 \bullet \cdot$. In the recent Scleroderma Lung Study, $1 \mathrm{cSSc}$ and dcSSc patients were indistinguishable with regard to their baseline pulmonary functions, but $\mathrm{lcSSc}$ patients presented with more extensive pulmonary fibrosis, possibly reflecting a delay in diagnosis and progression of lung disease prior to study entry $[30 \bullet \cdot$. The rate of progression of SILD is similar in $\mathrm{lcSSc}$ and dcSSc patients after adjustment for baseline differences in the degree of pulmonary fibrosis. Given that baseline forced vital capacity and pulmonary fibrosis scores were the most important independent predictors of the decline in pulmonary function over time in Scleroderma Lung Study patients, all SSc patients should be evaluated carefully for lung involvement irrespective of disease extent [29].

Criteria have been developed for scoring the severity and extent of SILD disease based on disease distribution, relative proportions of reticulations and GGO, and presence of fibrosis on CT scans at one or more levels throughout the
Fig. 1 Coronal and sagittal chest $\mathrm{CT}$ reconstructions (lung window) demonstrate the classic appearance of early lung involvement in scleroderma interstitial lung disease. Initially subtle alterations of increased ground glass opacity and accentuated reticular markings (a and $\mathbf{b}$ ) that affect the peripheral, posterior, and basilar portions of the lungs
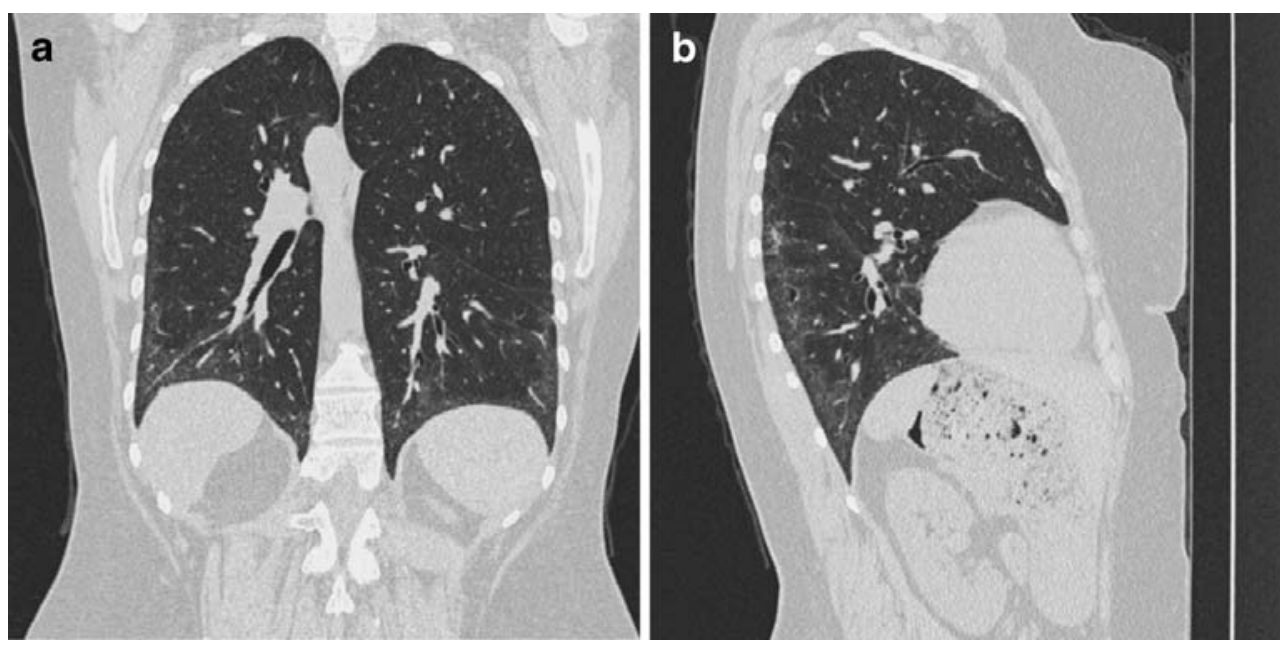
Fig. 2 Coronal and sagittal chest CT reconstructions (lung window) demonstrate the classic appearance of scleroderma interstitial lung disease in patients with progressive disease, showing extensive architectural distortion due to progressive pulmonary fibrosis (a and $\mathbf{b})$
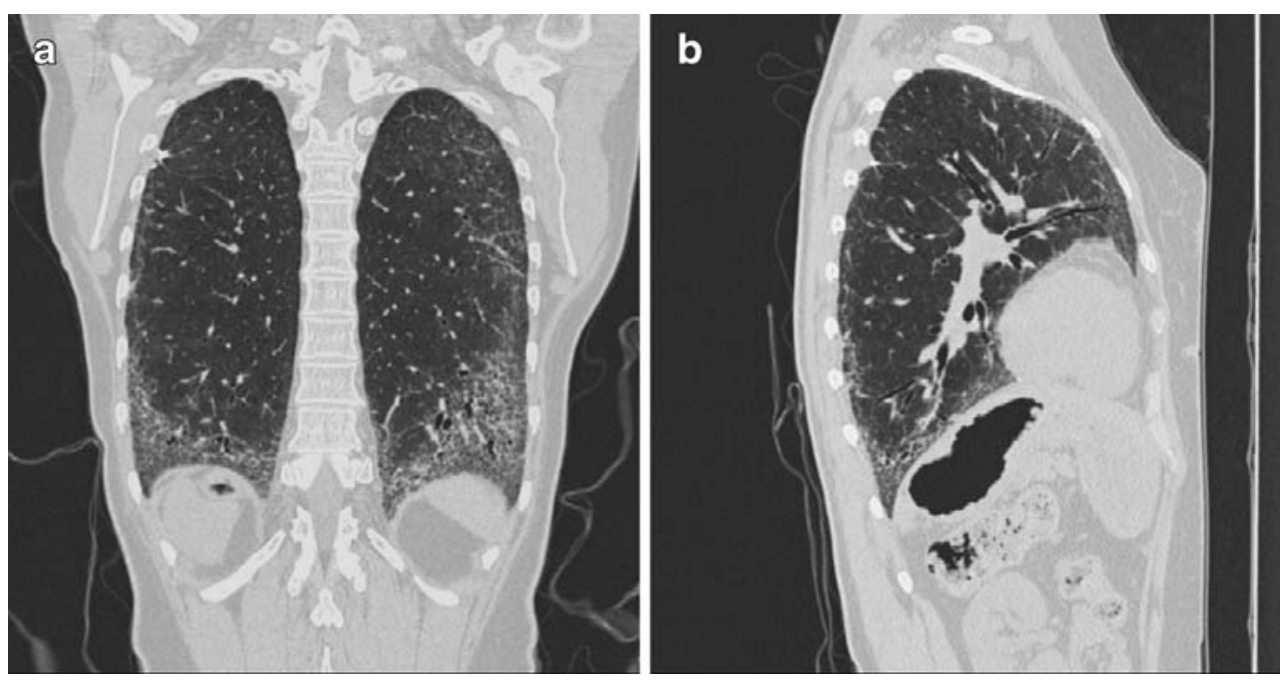

chest. These can be based on visual or computer-based quantification schema. Visual scoring systems typically divide each lung into three zones (lung apex to aortic arch, aortic arch to inferior pulmonary veins, inferior pulmonary veins to lung bases), and the extent of lung abnormality in each zone is scored on a scale of $0-4(0=$ absent; $1=1 \%-$ $25 \% ; 2=26 \%-50 \% ; 3=51 \%-75 \% ; 4=76 \%-100 \%$ ) [31]. More recently, a simplified scoring system based on whether more or less than $25 \%$ of the lung is affected has been suggested [32••]. Computer-based approaches also have been investigated and are based on measurement of the density or texture features of each pixel and assignment of a measure of the amount of abnormal lung tissue present. Computer-based models correlate well with visual scoring techniques for the detection of fibrosis (area under the curve $=0.86$ ) and with the assessment of extent of disease (area under the curve $=$ 0.96 for estimating lung involvement $>25 \%$ ) without the intrareader variation encountered with visual scoring [33].

\section{Prediction}

HRCT scanning has been used to predict outcome in addition to characterizing the nature and extent of SILD [34-36]. The absence of lung disease at an initial CT evaluation is a superior predictor of excellent long-term prognosis with regard to SILD. In a serial HRCT study of $90 \mathrm{SSc}$ patients, 40 had no evidence of pulmonary fibrosis on an initial scan; of these 40, 35 (88\%) continued to have a normal HRCT scan at follow-up for an average of 5 years [15]. Because many SSc patients have limited pulmonary fibrosis that may not necessarily progress, the decision to start treatment is often a clinical challenge. The estimation of disease extent (using HRCT) and severity (using PFT) is pivotal. Careful prognostic evaluation, including the staging of disease severity and the definition of longitudinal disease behavior (by serial imaging and PFT), is central to the formulation of a logical management plan [21].

It is now well-established that GGO is not indicative of active inflammation and likely represents microscopic pulmonary fibrosis that is below the resolution of CT. Previously, the presence of GGO in the absence of associated architectural distortion was thought to represent underlying inflammation, or "alveolitis," and to predict a high likelihood of reversible lung disease in SSc, based on studies in other diffuse lung diseases [13]. Recent studies have shown that at best, a weak relationship between GGO and abnormal bronchoalveolar lavage evidence for active lung inflammation [37]. Furthermore, the limitations of GGO as a predictive marker of active or reversible inflammation have been reinforced by longitudinal studies of CT change with and without treatment. When reticular interstitial abnormalities are present, as in most SSc cases, regression of disease on $\mathrm{CT}$ occurs in only a minority of patients in the short term [38]. In the long term, GGO usually progresses to overt fibrosis [16]. In a recent serial study of 41 SSc patients, GGO that occurred in two thirds of the cases regressed in only 5\% during the following 2 years and was often resistant to treatment [38]. Thus, the postulated relationship between GGO and active inflammation/alveolitis in SILD has been largely discredited.

More recently, the findings of fibrosis on HRCT scans of patients with SILD have been shown to be a useful predictor of the progression of fibrosis when untreated and of a favorable response to treatment with cyclophosphamide compared with placebo [36]. The extent of pulmonary fibrosis on $\mathrm{CT}$ also has played a key role in determining the prognosis of patients with $\mathrm{SSc}[32 \bullet \cdot]$. In a study of 215 patients, those with more extensive disease on HRCT (i.e., abnormalities involving $>20 \%$ of the lung volume) had strikingly higher mortality and rapid decline of lung function, whereas patients with less than $20 \%$ 
abnormality had no increase in long-term mortality compared with SSc patients with a normal HRCT [32••]. To overcome the difficulties in formally scoring disease extent on HRCT in routine practice, the authors proposed a simple semiquantitative staging system of limited and extensive disease, in which CT evaluation and forced vital capacity values are integrated.

\section{Treatment Outcomes}

Experience is limited with the use of HRCT as an outcome measure in therapeutic trials. HRCT scanning has been used only infrequently in a prospective, systematic manner as an outcome parameter for assessing therapeutic responses in patients with SILD [39-43]. The validity of serial CT studies is also compromised by the difficulty in achieving exact anatomic comparability between initial and follow-up CT scans, which is due to inherent differences in HRCT slice acquisitions. Low-dose volume (spiral) acquisition CT techniques may overcome this problem, and several studies are under way. In contrast, when anatomic comparability is achieved, it may be difficult to ascertain the clinical significance of minor $\mathrm{CT}$ changes that are confined to limited lung regions. The recent serial study of Launay et al. [15] was limited by their definition of change in lung involvement by more or less than $50 \%$ of the lung volume. This system may fail to identify significant change in many cases in which the arbitrary threshold of $50 \%$ is not crossed. In a more recent study, when readers graded SILD on serial HRCT as worsened, unchanged, or improved, there was an excellent correlation among CT changes, patient outcome, and treatment efficacy that was only marginal as suggested by conventional pulmonary function criteria [43].

\section{Other Significant Thoracic Diseases}

$\mathrm{PH}$, defined as pulmonary artery pressure greater than $35 \mathrm{~mm}$ $\mathrm{Hg}$ as estimated by transesophageal echocardiography, affects about $20 \%$ (range, $6 \%-60 \%$ ) of patients with $\operatorname{SSc}[2,3]$ and is a major cause of SSc-related death [44••]. When complicated by $\mathrm{PH}$, systemic sclerosis has a very poor prognosis. About half of patients with $\mathrm{PH}$ develop the disease within 5 years of the SSc diagnosis [44••]. Primary cardiac involvement from SSc may smolder for years before manifesting as overt cardiomyopathy, pericardial disease, or conduction abnormalities [45]. SSc heart disease has a poor prognosis and may be related to excessive deposition of abnormal collagen that impairs myocardial contractility, and to ischemia from coronary vasculopathy [46].

Scleroderma involves the gastrointestinal tract in up to $90 \%$ of patients, and fibrosis and atrophy may result in motility disorders. Esophageal involvement is often an early and asymptomatic manifestation of SSc. A dilated esophagus containing fluid, gas, and/or debris in the setting of SSc reflects esophageal involvement and may help differentiate ILD due to connective tissue disease from other etiologies (Fig. 3). Asymptomatic esophageal dilatation and esophageal dysmotility are detected in up to $80 \%$ of patients [47]. Gastroesophageal reflux may play a role in the development and/or progression of SILD [48, 49]. Early detection and treatment of esophageal involvement may forestall complications, which include chronic reflux, aspiration pneumonia, Barrett's esophagus, and esophageal stricture and malignancy.

The presence of mediastinal lymph node enlargement in patients with SILD is relatively higher than other causes of ILD and has been reported in up to $60 \%$ of cases [50]. Nodal enlargement typically corresponds to reactive change on histopathology when biopsy is performed.

\section{Conclusions}

Scleroderma is a systemic disorder of connective tissues and vasculopathy that involves multiple organ systems, typically the skin, esophagus, and lungs. Symptoms of ILD may frequently overlap with those of $\mathrm{PH}$, aspiration pneumonia, and cardiomyopathy. As patients with limited and diffuse cutaneous scleroderma may exhibit progressive and extensive pulmonary fibrosis, all scleroderma patients

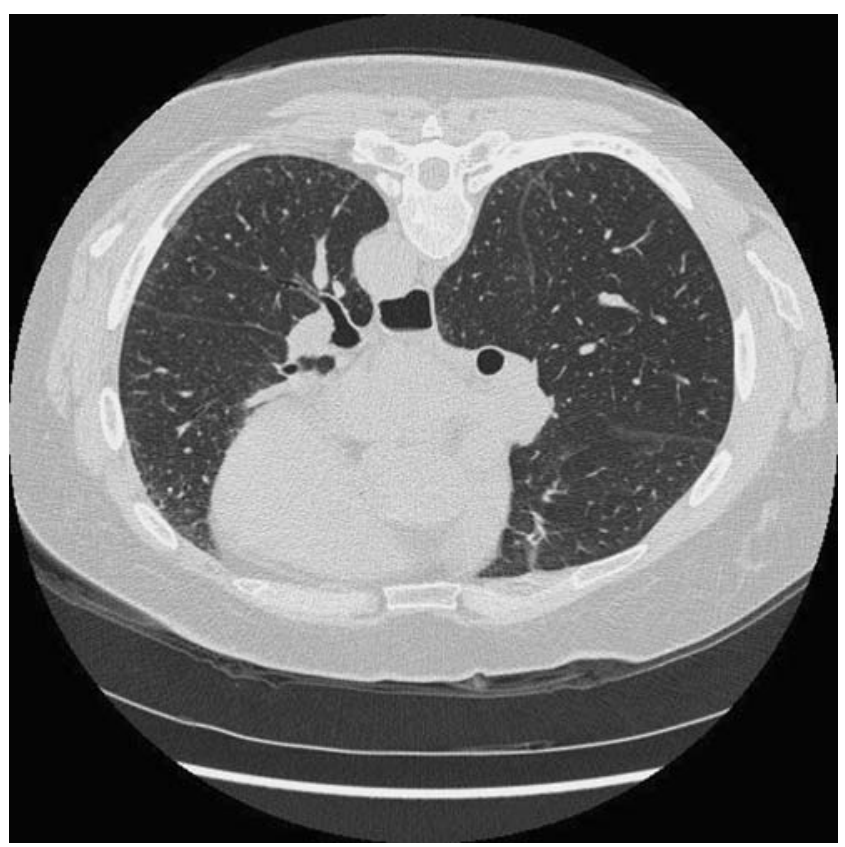

Fig. 3 Esophageal dilatation with retained debris is an early and frequent manifestation of scleroderma and may help differentiate interstitial lung disease due to connective tissue disease from that of other etiologies 
should be evaluated for lung involvement. Histopathologic assessment of SILD typically represents overlapping features with NSIP and UIP.

HRCT, in conjunction with PFT results, plays a critical role in the detection and treatment of SILD and in the prediction of outcomes. Visual scoring and computer-based HRCT techniques are comparable in detecting and grading disease severity and progression. On CT, SILD typically manifests as GGO and accentuated reticulations that may progress to pulmonary fibrosis. GGO was recently discredited as a marker of active inflammation and reversible lung injury.

Acknowledgment Dr. Goldin has received funding from the National Institutes of Health.

Disclosure Dr. Goldin is the director of MedQIA. No other potential conflicts of interest relevant to this article were reported.

Open Access This article is distributed under the terms of the Creative Commons Attribution Noncommercial License which permits any noncommercial use, distribution, and reproduction in any medium, provided the original author(s) and source are credited.

\section{References}

Papers of particular interest, published recently, have been highlighted as:

•. Of major importance

1. Kreig T, Takehara K: Skin disease: a cardinal feature of systemic sclerosis. Rheumatology (Oxford) 2009, 48(Suppl 3): iii14-iii18.

2. Silver RM, Miller KS: Lung involvement in systemic sclerosis. Rheum Dis Clin North Am 1990, 16:199-216.

3. Ramirez A, Varga J: Pulmonary arterial hypertension in systemic sclerosis: clinical manifestations, pathophysiology, evaluation, and management. Treat Respir Med 2004, 3:339-352.

4. Gilliland BC: Systemic sclerosis (scleroderma). In Harrison's Principles of Internal Medicine, edn 15. Edited by Braunwald E, Fauci AS, Kasper DL, et al. New York: McGraw-Hill, 2001.

5. Arroliga AC, Podell DN, Matthay RA: Pulmonary manifestations of scleroderma. J Thorac Imaging 1992, 7:30-45.

6. Ungerer RG, Tashkin DP, Furst D, et al.: Prevalence and clinical correlates of pulmonary arterial hypertension in progressive systemic sclerosis. Am J Med 1983, 75:65-74.

7. Mukerjee D, St George D, Coleiro B, et al.: Prevalence and outcome in systemic sclerosis associated pulmonary arterial hypertension: application of a registry approach. Ann Rheum Dis 2003, 62:1088-1093.

8. Steen VD, Conte C, Owens GR, Medsger TA Jr: Severe restrictive lung disease in systemic sclerosis. Arthritis Rheum 1994, 37:12831289 .

9. White B, Moore WC, Wigley FM, et al.: Cyclophosphamide is associated with pulmonary function and survival benefit in patients with scleroderma and alveolitis. Ann Intern Med 2000, 132:947-954.
10. Bianchi FA, Bistue AR, Wendt VE, et al.: Analysis of twentyseven cases of progressive systemic sclerosis and a review of the literature. J Chronic Dis 1966, 19:953-977.

11. Schurawitzki H, Stiglbauer R, Graninger W, et al.: Interstitial lung disease in progressive systemic sclerosis: high resolution CT versus radiography. Radiology 1990, 176:755-759.

12. Swensen SJ, Aughenbaugh GL, Myers JL: Diffuse lung disease: diagnostic accuracy of $\mathrm{CT}$ in patients undergoing surgical biopsy of the lung. Radiology 1997, 205:229-234.

13. Remy-Jardin M, Remy J, Wallaert B, et al.: Pulmonary involvement in progressive systemic sclerosis: sequential evaluation with CT, pulmonary function tests, and bronchoalveolar lavage. Radiology 1993, 188:499-506.

14. Diot E, Boissinot E, Asquier E, et al.: Relationship between abnormalities on high-resolution $\mathrm{CT}$ and pulmonary function in systemic sclerosis. Chest 1998, 114:1623-1629.

15. Launay D, Remy-Jardin M, Michon-Pasturel U, et al.: High resolution computed tomography in fibrosing alveolitis associated with systemic sclerosis. J Rheumatol 2006, 33:1789-1801.

16. Desai SR, Veeraraghavan S, Hansell DM, et al.: CT features of lung disease in patients with systemic sclerosis: comparison with idiopathic pulmonary fibrosis and nonspecific interstitial pneumonia. Radiology 2004, 232:560-567.

17. Goldin JG, Lynch DA, Strollo DC, et al.: High resolution CT findings in scleroderma-related lung diseases: findings from Scleroderma Lung Study. Chest 2008, 134:358-367.

18. Kim EA, Johkoh T, Lee KS, et al.: Interstitial pneumonia in progressive systemic sclerosis: serial high-resolution CT findings with functional correlation. J Comput Assist Tomogr 2001, 25:757-763.

19. Fujita J, Yoshinouchi T, Ohtsuki Y, et al.: Non-specific interstitial pneumonia as pulmonary involvement of systemic sclerosis. Ann Rheum Dis 2001, 60:281-283.

20. Muller NL, Colby TV: Idiopathic interstitial pneumonias: highresolution CT and histologic findings. Radiographics 1997, 17:1016-1022.

21. Latsi PI, Wells AU: Evaluation and management of alveolitis and interstitial lung disease in scleroderma. Curr Opin Rheumatol 2003, 15:748-755.

22. Lynch DA: Nonspecific interstitial pneumonia: evolving concepts. Radiology 2001, 221:583-584.

23. Bouros D, Wells AU, Nicholson AG, et al.: Histopathologic subsets of fibrosing alveolitis in patients with systemic sclerosis and their relationship to outcome. Am J Respir Crit Care Med 2002, 165:1581-1586.

24. Kim DS, Yoo B, Lee JS, et al.: The major histopathologic pattern of pulmonary fibrosis in scleroderma is nonspecific interstitial pneumonia. Sarcoidosis Vasc Diffuse Lung Dis 2002, 19:121-127.

25. Minai OA, Dweik RA, Arroliga AC: Manifestations of scleroderma pulmonary disease. Clin Chest Med 1998, 19:713-731.

26. Hunninghake GW, Lynch DA, Galvin JR, et al.: Radiologic findings are strongly associated with a pathologic diagnosis of usual interstitial pneumonia. Chest 2003, 124:1215-1223.

27. Colby TV, Carrington CB: Interstitial lung disease. In Pathology of the Lung. Edited by Thurlbeck WM, Churg AM. New York: Thieme Medical Publishers; 1995:589-737.

28. Piper WN, Helwig EB: Progressive systemic sclerosis: visceral manifestations in generalized scleroderma. AMA Arch Derm 1955, 72:535-546.

29. Fischer A, Swigris JJ, Groshong SD, et al.: Clinically significant interstitial lung disease in limited scleroderma: histopathology, clinical features, and survival. Chest 2008, 134:601-605.

30. •• Clements PJ, Roth MD, Elashoff R, et al.: Scleroderma Lung Study (SLS): differences in the presentation and course of patients with limited versus diffuse systemic sclerosis. Ann Rheum Dis 2007, 66:1641-1647. This was a prospective treatment study of 
cyclophosphamide in patients with moderate to severe lung involvement showing that there is similar involvement of the lungs in localized, limited, and diffuse patient groups.

31. Kazerooni EA, Martinez FJ, Flint A, et al.: Thin-section CT obtained at $10-\mathrm{mm}$ increments versus limited three-level thinsection CT for idiopathic pulmonary fibrosis: correlation with pathologic scoring. AJR Am J Roentgenol 1997, 169:977-983.

32. • Goh NS, Desai SR, Veeraraghavan S, et al.: Interstitial lung disease in systemic sclerosis: a simple staging system. Am J Respir Crit Care Med 2008, 177:1248-1254. This was a landmark study demonstrating a predictive value to estimating disease extent on CT and patient survival.

33. Kim HJ, Li G, Gjertson D, et al.: Classification of parenchymal abnormality in scleroderma lung using a novel approach to denoise images collected via a multicenter study. Acad Radiol 2008, 15:1004-1016.

34. Wells AU, Rubens MB, du Bois RM, Hansell DM: Serial CT in fibrosing alveolitis: prognostic significance of the initial pattern. AJR Am J Roentgenol 1993, 161:1159-1165.

35. Wells AU: High-resolution computed tomography and scleroderma lung disease. Rheumatology (Oxford) 2008, 47(Suppl 5):v59-v61.

36. Tashkin DP, Elashoff R, Clements PJ, et al.: Cyclophosphamide versus placebo in scleroderma lung disease. N Engl J Med 2006, 354:2655-2666.

37. Strange C, Bolster MB, Roth MD, et al.: Bronchoalveolar lavage and response to cyclophosphamide in scleroderma interstitial lung disease. Am J Respir Crit Care Med 2008, 177:91-98.

38. Shah RM, Jimenez S, Wechsler R: Significance of ground-glass opacity on HRCT in long-term follow-up of patients with systemic sclerosis. J Thorac Imaging 2007, 22:120-124.

39. Giacomelli R, Valentini G, Salsano F, et al.: Cyclophosphamide pulse regimen in the treatment of alveolitis in systemic sclerosis. J Rheumatol 2002, 29:731-736.

40. Griffiths B, Miles S, Moss H, et al.: Systemic sclerosis and interstitial lung disease: a pilot study using pulse intravenous methylprednisolone and cyclophosphamide to assess the effect on high resolution computed tomography scan and lung function. J Rheumatol 2002, 29:2371-2378.

41. Pakas I, Ioannidis JPA, Malagari K, et al.: Cyclophosphamide with low or high dose prednisolone for systemic sclerosis lung disease. J Rheumatol 2002, 29:298-304.

42. Davas EM, Peppas C, Maragou M, et al.: Intravenous cyclophosphamide pulse therapy for the treatment of lung disease associated with scleroderma. Clin Rheumatol 1999, 18:455-461.

43. Goldin J, Elashoff R, Kim HJ, et al.: Treatment of sclerodermainterstitial lung disease with cyclophosphamide is associated with less progressive fibrosis on serial thoracic high-resolution CT scan than placebo. Chest 2009, 136:1333-1340.

44. •- McLaughlin V: Pulmonary arterial hypertension: the most devastating vascular complication of systemic sclerosis. Rheumatology (Oxford) 2009, 48(Suppl 3):iii25-iii31. This is an excellent article demonstrating the role of $\mathrm{PH}$ in the morbidity and mortality of SILD.

45. Kahan A, Coghlan G, McLaughlin V: Cardiac complications of systemic sclerosis. Rheumatology (Oxford) 2009, 48(Suppl 3): iii45-iii48.

46. Vonk Noordegraaf A, Naeije R: Right ventricular function in scleroderma-related pulmonary hypertension. Rheumatology (Oxford) 2008, 47(Suppl 5):v42-v43.

47. Lahcene M, Oumnia N, Matougui N, et al.: Esophageal dysmotility is scleroderma: a prospective study of 183 cases. Gastroenterol Clin Biol 2009, 33:466-469.

48. Tobin RW, Pope CE 2nd, Pellegrini CA, et al.: Increased prevalence of gastroesophageal reflux in patients with idiopathic pulmonary fibrosis. Am J Respir Crit Care Med 1998, 158:1804-1808.

49. Marie I, Dominique S, Levesque H, et al.: Esophageal involvement and pulmonary manifestations in systemic sclerosis. Arthritis Rheum 2001, 45:346-354.

50. Bhalla M, Silver RM, Shepard JA, McLoud TC: Chest CT in patients with scleroderma: prevalence of asymptomatic esophageal dilatation and mediastinal lymphadenopathy. AJR Am J Roentgenol 1993, 161:269-272. 